\title{
Constrained Optimization on Hierarchies and Braids of Partitions
}

\author{
Jean Serra ${ }^{1}$ and B Ravi Kiran ${ }^{2}$ \\ 1 Université Paris-Est, A3SI-ESIEE LIGM \\ jean.serra@esiee.fr \\ 2 Centre de robotique, MINES ParisTech, PSL-Research University, \\ ravi.kiran@mines-paritech.fr
}

\begin{abstract}
This theoretical paper provides a basis for the optimality of scale-sets by Guigues [6] and the optimal pruning of binary partition trees by Salembier-Garrido [11]. They extract constrained-optimal cuts from a hierarchy of partitions. Firstly, this paper extends their results to a larger family of partitions, namely the braid [9]. Secondly, the paper shows the dependence of valid constraint function values and multiplier values in a Lagrangian optimization framework. Lastly, but most importantly, it also proposes the energetic order and energetic lattice based solutions for the constraint optimization problem. This approach operates on a partition based constraint thus ensuring the existence of a valid multiplier and constraint value.
\end{abstract}

Keywords: Hierarchies, Lagrange, Optimization, Lattice

\section{Introduction}

In this theoretical paper ${ }^{3}$ we aim to first demonstrate that the optimal cuts on hierarchies or $\lambda$-cuts in the sense of Guigues [6] and Salembier et al.[11], only provide an upper bound on the minimum energy to the original constrained optimization problem on hierarchies. We show that the choice of a suitable Lagrange multiplier $\lambda$ in fact provides a solution to the perturbed problem first stated in Everett's theorem [5]. Further in a fundamental contribution we demonstrate how the constrained optimization problem in the Lagrange sense can be solved using the energetic lattice [7], by replacing the numerical constraints by partition based constraint. We start with a quick review of notation and definitions.

\subsection{Definitions}

We denote a partition of space $E$ by $\pi$ and a partial partition(p.p.) [10] of subset $S \subseteq E$ by $\pi(S)$. The family of all partitions of $E$ is denoted by $\Pi(E)$, while that

\footnotetext{
${ }^{3}$ Please refer to accompanying paper [9] for notions of braids, energetic ordering, energetic lattice, singularity and $h$-increasingness. This work was partly funded by the French ANR-2010-BLAN-0205-03 program KIDICO.
} 
of partial partitions by $\mathcal{D}(E)$ ). A hierarchy of partitions (HOP) is a finite chain of partitions $H=\left\{\pi_{i}, i \in[0, n]\right\}$, with $\pi_{i} \leq \pi_{j}, i<j$, where $\leq$ stands for the refinement ordering. The minimal element $\pi_{0}$ of $H$ is called the leaves partition, while the maximal element is the one class partition $\{E\}$, called the root. A cut of hierarchy $H$ is a partition of $E$ whose elements are composed of classes in $H$. The set of all cuts of $H$ is $\Pi(E, H)$. A braid $B$ is a family of partitions $B$ where the pairwise refinement supremum of any two partial partitions is a cut of some hierarchy $H$; i.e. belongs to $\Pi(E, H)[9]$.

An energy $\omega: \mathcal{D} \rightarrow \mathbb{R}^{+}$is a non-negative function that is defined on the family of partial partitions. The energy of a partition or partial partition is obtained by the composition of energies either by addition, supremum or other laws [9] of its constituent classes, $\omega(\pi(S))=\sum_{a \sqsubset \pi(S)} \omega(a)$, though these might not be the only way. The energy $\omega$ is said to be singular when for any p.p. $\pi(S)$ we have $\omega(\{S\}) \neq \omega(\pi(S)), S \subseteq E$. It is said to be $h$-increasing when

$$
\omega(\pi(S)) \leq \omega\left(\pi^{\prime}(S)\right) \Rightarrow \omega\left(\pi(S) \sqcup \pi_{0}\right) \leq \omega\left(\pi^{\prime}(S) \sqcup \pi_{0}\right), \quad \forall S \subseteq E
$$

where $\sqcup$ indicates the concatenation of any p.p $\pi_{0}$ with support that is disjoint with $S$. A $h$-increasing energy becomes strict when the inequality $\leq$ becomes $<$.

Now the optimal cut in [4], [11], [6], is calculated by aggregating local optima. The local optimum at class $S$ either choses the parent $\{S\}$, or the disjoint union of the optimums over the its children as shown in equation 2.

$$
\pi^{*}(S)= \begin{cases}\{S\}, & \text { if } \omega(S) \leq \operatorname{comp}\left(\omega\left(\pi^{*}(a)\right)\right), a \in \pi(S) \\ \bigsqcup_{a \in \pi(S)} \pi^{*}(a), & \text { otherwise }\end{cases}
$$

The solution to the dynamic program in equation (2), when aggregated for all $S \in H$, following a lexicographic order gives the optimal cut $\pi^{*}$. One should also note that the composition $\operatorname{comp}(\cdot)$ is performed by addition, or supremum, or many other laws that preserves $h$-increasingness. It is shown in [7] that this optimal cut is the minimal element of an energetic lattice. We reproduce the theorem in [7]:

Theorem 1. Let $\Pi$ be a family of partitions of $E$, and let $\pi_{1}, \pi_{2} \in \Pi$. Given an energy $\omega$, the partition $\pi_{1}$ is said to be less energetic than $\pi_{2}$, and one writes $\pi_{1} \preceq_{\omega} \pi_{2}$ when in each class of $\pi_{1} \vee \pi_{2}$ the energy of the partial partition of $\pi_{1}$ is smaller or equal to that of $\pi_{2:}$ :

$$
\pi_{1} \preceq_{\omega} \pi_{2} \quad \Leftrightarrow \quad\left\{S \in \pi_{1} \vee \pi_{2} \Rightarrow \omega\left(\pi_{1} \sqcap\{S\}\right) \leq \omega\left(\pi_{2} \sqcap\{S\}\right)\right\}
$$

The relation $\preceq_{\omega}$ is an ordering relation for all singular energies $\omega$, called energetic ordering, if and only if the family $\Pi$ is the set $\Pi(\omega, E, B)$ of all cuts of a braid $B$.

The set $\Pi(\omega, E, B)$ forms a complete lattice for the energetic ordering $\preceq_{\omega}$.

This theorem described an energetic ordering and thus an energetic lattice [9], which models the dynamic program to obtain the optimal cut. We will use this 
lattice structure for the constrained optimization problem on HOP and braids further on in this paper.

\subsection{Constrained Optimization on Hierarchies}

A lattice structure has been developed in [9], [7] for the dynamic program based minimization of any general non parametrized energy. We now concentrate on the constrained optimization problem, which is achieved by the unconstrained minimization of a Lagrangian function, as is the case with Guigues and Salembier. Consider the constrained optimization problem:

$$
\underset{\pi \in \Pi(E, H)}{\operatorname{minimize}} \sum_{S \in \pi} \omega_{\varphi}(S) \quad \text { subject to } \quad \sum_{S \in \pi} \omega_{\partial}(S) \leq C
$$

The corresponding Lagrangian can now be written as:

$$
\omega(\pi, \lambda)=\sum_{S \in \pi} \omega_{\varphi}(S)+\lambda \cdot\left(\omega_{\partial}(S)-C\right)
$$

where $\pi \in \Pi(E, H)$ is a cut from HOP $H$. The objective function being minimized is denoted by $\omega_{\varphi}$, the constraint function by $\omega_{\partial}$, while $C$ is an imposed constraint function value. These energies hold on all partial partitions $\omega_{\varphi}, \omega_{\partial}: \mathcal{D} \rightarrow \mathbb{R}$ of the working space $E$. In (5) the multiplier $\lambda$ is scalar for the sake of simpler notation and pedagogy. This can always extend to vector multiplier and constraint functions.

\section{Guigue's $\lambda$-cuts are upper bounds}

We illustrate in Figure 1 a tree with its classes, objective function being minimized, constraint function, as well as the $\lambda$ or scale function values as defined by Guigues [6]. The objective $\omega_{\varphi}$ is chosen to be super-additive while constraint $\omega_{\partial}$ is sub-additive, as in [6]. Now the family of Lagranians is $\left\{\omega(\lambda)=\omega_{\varphi}+\lambda \omega_{\partial}, \lambda \geq 0\right\}$.

Energies are composed additively here, i.e. $\omega(\pi(S))=\sum_{a \in \pi(S)} \omega(a)$. Further when we have equal parent and child energies, we pick the parent, like in [6].

The $\lambda$-cut denoted by $\pi^{*}(\lambda)$ are cuts with least $\omega_{\varphi}$, given multiplier $\lambda$. There are three such cuts, for three different values of the multiplier calculated by the scale function defined by Guigues $[6] \lambda=-\Delta \omega_{\varphi} / \Delta \omega_{\partial}$.

Counter Example: Following Guigues and Salembier (as well as Casselles et al. [2]) we search for the cut with the smallest $\lambda$, or $\lambda$-cut, which satisfies an input constraint value here set to $C=7.5$. Here the $\lambda$-cut with $\omega_{\varphi}\left(\pi^{*}(\lambda=3.5)\right)=15$ and constraint $\omega_{\partial}\left(\pi^{*}(\lambda=3.5)\right)=6$ is the optimal $\lambda$-cut satisfying constraint. We now consider other cuts, which are not $\lambda$-cuts: say $\pi=(g, c, d, k)$ with energy $\omega(\pi, \lambda)=11.5+7 \lambda$. The cut $\pi$ obviously provides a better minimum than the minimal $\lambda$-cut $(g, h, k)$ since $\omega_{\partial}(\pi)=7$, which is below the constraint $C=7.5$, for an objective value $\omega_{\varphi}(\pi)=11.5$, which also is smaller than the objective function $\left.\omega_{\varphi}\left(\pi^{*}(\lambda=3.5)\right)=15\right)$. What is worse is that there are 

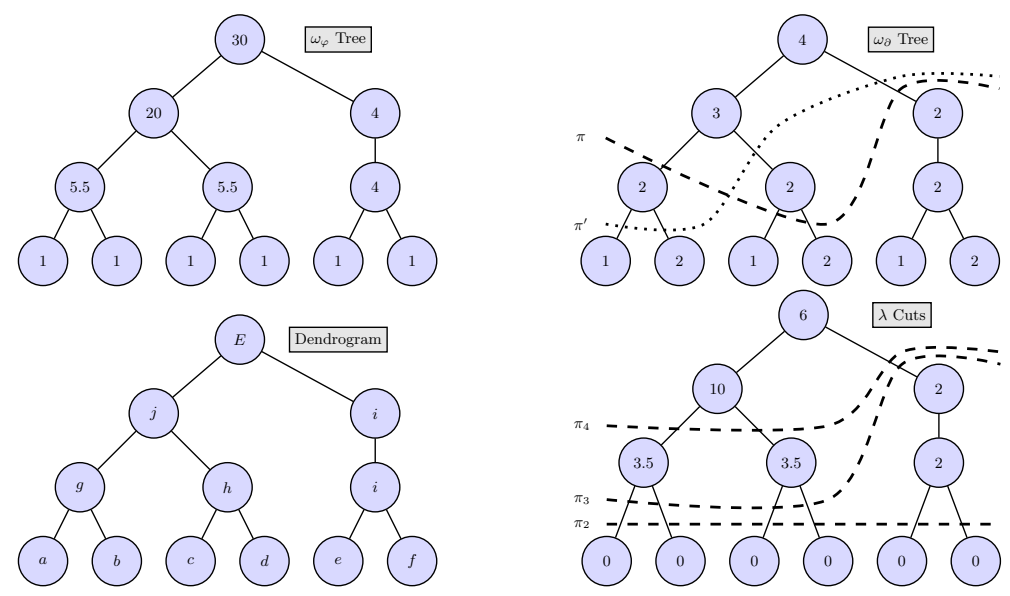

Fig. 1. Bottom Left, a hierarchy $H$ with classes. The two trees in the top row, indicate the two energies $\left(\omega_{\varphi}, \omega_{\partial}\right)$ associated with the corresponding classes. $\pi$ and $\pi^{\prime}$ are two cuts of $H$. Bottom right, in the nodes, we depict the $\lambda$ values by equating parent and child energies, whose level sets give the minimal cuts w.r.t. the $\omega_{\lambda}$. They are depicted in the $\lambda$-tree for $\lambda=2,3.5,4$ as $\pi_{2}, \pi_{3}, \pi_{4}$. The $\lambda$ values for the leaves are assumed to be 0 , though in case of Breiman et al. [4] $\lambda$ for the leaf classes are set to $\infty$ to avoid over-fitting.

two such different cuts $\pi=(g, c, d, k)$ and $\pi^{\prime}=(a, b, h, k)$, that have the same constraint and objective values, $\omega_{\partial}\left(\pi^{\prime}\right)=7$ and $\omega_{\varphi}\left(\pi^{\prime}\right)=11.5$. Thus there are several constrained minimal cuts for the energy $\omega_{\varphi}$, and none of them are obtained from the sequence of $\lambda$-cuts $\pi^{*}(\lambda)$ ! And we cannot take their infimum $\pi \wedge \pi^{\prime}=(a, b, c, d, k)$ because $\omega_{\partial}\left(\pi \wedge \pi^{\prime}\right)=8$, which is above the constraint $C=7.5$. The plot of the different energies of the $\lambda$-cuts with the constraint are shown in Figure 2.

Observations: For an imposed cost $\omega_{\partial}(\pi) \leq C$ one is not assured of the existence of a corresponding multiplier value $\lambda$. The family of $\lambda$-cuts $\left\{\pi^{*}(\lambda)\right.$, $\lambda \in \overline{\mathbb{R}}\}$ is not complete to describe all possible constraint values. A cut that minimizes $\omega_{\varphi}$ may not belong to the $\lambda$-cuts $\left\{\pi^{*}(\lambda)\right\}$. One may also remark that the dual problem still remains a combinatorial problem. As we know from convex optimization [3], given a multiplier, the dual Lagrangian serves as an upper bound on the optimum corresponding to the primal Lagrangian. In our words, $\pi^{*}\left(\lambda^{*}\right)$ is only the upper-bound of the constrained minimal cuts. Furthermore the values of $\lambda$ can be discrete, real, or rational, while still lacking a $C \rightarrow \lambda$ constraint-multiplier map. Finally, one can note that uniqueness is lost, even when $\omega_{\varphi}$ is strictly $h$-increasing. 


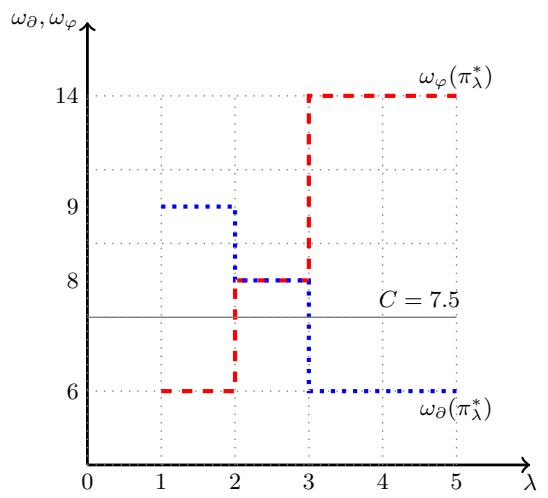

Fig. 2. For $2<\lambda<3$ the minimal cut is $(a, b, c, d, i)$ and $\omega_{\partial}=8$, for $\lambda \geq 3$ the minimal cut is $(g, h, i)$ and $\omega_{\partial}=6$, i.e. $\omega_{\partial}$ is never equal to the cost $C=7.5$ at any time.

\section{Everett's Theorem}

Everett's seminal paper [5] studies resource allocation problem by a choosing an optimal Lagrangian parameter. In literature one of its earliest uses appears in source-coding by its usage by Shoham-Gersho [12] to study variable rate set quantizers. The function $f$ is an objective being minimized and $g, h$ are inequality and equality constraints, which are three real valued functions defined over an arbitrary abstract set $X . X$ need not be topological. Neither do we require continuity, derivation, or convexity of the functions $f, g, h$.

Theorem 2. Given the Lagrangian function, with multipliers $\lambda, \mu$

$$
\min _{\boldsymbol{x} \in X}\{f(x)+\mu g(x)+\lambda h(x)\}
$$

the solution $\bar{x}(\lambda)$ to this unconstrained minimization problem, is also an optimal solution to perturbed primal problem, namely

$$
\begin{array}{ll}
\underset{\boldsymbol{x} \in X}{\operatorname{minimize}} & f(\boldsymbol{x}) \\
\text { subject to } & g(\boldsymbol{x}) \leq g(\overline{\boldsymbol{x}}(\boldsymbol{\lambda})) ; h(\boldsymbol{x})=h(\overline{\boldsymbol{x}}(\boldsymbol{\lambda})) ;
\end{array}
$$

For Guigues and Salembier's problem, this can be restated as:

Theorem 3. Given the multiplier $\lambda \in \mathbb{R}$ and the Lagrangian function,

$$
\min _{\pi \in \Pi(E, H)}\left\{\sum_{S \in \pi} \omega_{\varphi}(S)+\lambda \sum_{S \in \pi} \omega_{\partial}(S)\right\}
$$

the solution $\bar{\pi}(\lambda)$ to this unconstrained minimization is also an optimal solution to perturbed primal problem: 


$$
\operatorname{minimize}_{\pi \in \Pi(E, H)}^{\operatorname{minim}} \quad \sum_{\pi} \omega_{\varphi}(S) \text { subject to } \quad \sum_{S \in \pi} \omega_{\partial}(S) \leq \sum_{S \in \bar{\pi}(\lambda)} \omega_{\partial}(S)
$$

Everett's theorem states the following: for any non-negative $\lambda$, if an unconstrained minimum of the Lagrangian function can be found, with solution $\bar{x}(\lambda)$ or $\bar{\pi}(\lambda)$, then this solution is also the solution to the constrained problem whose constraints are, in fact, the amount of each resource expended in achieving the unconstrained solution. This implies that the constraints are set by choosing the $\lambda$ parameter. Any arbitrary set of non-negative $\lambda$ 's works here, notably causing the original constraint optimization problem to be unknown, and is only to be defined once the Lagrangian's solutions are determined.

\section{Constrained optimization by energetic lattices}

We now present the energetic lattice framework for constrained optimization. We shall first reformulate the minimization of the Lagrangian optimization using the energetic lattice. Further on we develop a constrained optimization model, where the constraint values a are based on refinement of partitions, as against the purely numerical order. We also introduce inf-modularity, which generalizes the sub-additivity of constraint function in Guigues [6], resulting in the important Theorem 4.

\subsection{Refinement and energetic lattices}

There are two types of lattices we refer to in this paper, refinement lattices and energetic lattices. The refinement lattices over a family of partitions, namely $\Pi(E)$ all partitions, $\Pi(E, H)$, partitions from a hierarchy $H$ and finally $\Pi(E, B)$ partitions from a braid $B$. Here $\Pi(E, H), \Pi(E, B)$ are sub-lattices of $\Pi(E)$. When we say two partitions are ordered, $\pi_{i} \leq \pi_{j}$, we refer to the refinement ordering.

Given this family of partitions, and any singular energy $\omega$ we can now consider the corresponding energetic order and lattice. Here, $\Pi_{\omega(\lambda)}$ is a based on the energetic ordering $\preceq_{\omega(\lambda)}$ w.r.t the Lagrangian $\omega(\pi, \lambda)$, of order $\preceq_{\omega(\lambda)}$. The minimal cut for this energetic lattice is $\pi^{*}(\lambda)=\curlywedge_{\omega(\lambda)}\{\pi, \pi \in \Pi\}$. The value of $\omega(\pi, \lambda)$ for a cut $\pi \in \Pi(E, B)$ is denoted by $\omega(\pi, \lambda)$, and that for the minimal cut by $\omega\left(\pi^{*}(\lambda)\right)$. Similarly we have the lattices $\Pi_{\omega_{\varphi}}$ and $\Pi_{\omega_{\partial}}$ for $\left(\preceq_{\omega_{\varphi}}, \omega_{\varphi}\right)$ and $\left(\preceq_{\omega_{\partial}}, \omega_{\partial}\right)$ energetic order-energy pairs, respectively. In these lattices the family of partitions under study are assumed to be cuts, either from the hierarchy or the braid under study.

\subsection{Inf-modularity}

Definition 1. An energy $\omega_{\partial}: \mathcal{D}(E) \rightarrow \mathbb{R}^{+}$is said inf-modular when for each p.p. $\pi$ of support $S \in \mathcal{P}(E)$ we have

$$
\omega_{\partial}(\{S\}) \leq \bigwedge\left\{\omega_{\partial}(a), a \sqsubseteq\{S\}\right\} .
$$


Inf-modularity provides a non-linear version of sub-additivity [1], where the former acts on partial partitions, while latter on general subsets of the space. An energy $\omega$ is sub-additive when, for any p.p. $\pi(S)$ of support $S$, the energy $\omega(S) \leq \sum_{T_{i} \in \pi(S)} \omega\left(T_{i}\right)$ for the sake of comparison. Since the energy $\omega$ is defined on partial partitions of $E$, we need to introduce the energy $\omega_{\partial}^{\prime}$ on sets such that $\omega_{\partial}^{\prime}(S)=\omega_{\partial}(\{S\})$. Then any extension $\omega_{\partial}$ of $\omega_{\partial}^{\prime}$ to the p.p. of $E$ which satisfies the following inequality is inf-modular.

$$
\omega_{\partial(S)}(\pi) \leq \sum_{j=1}^{j=p} \omega_{\partial}\left(\left\{T_{j}\right\}\right)=\sum_{j=1}^{j=p} \omega_{\partial}^{\prime}\left(T_{j}\right),
$$

Conversely, the restriction $\omega_{\partial}^{\prime}$ to sets of an inf-modular energy $\omega_{\partial}$ is sub-additive [8]. For example, in a partition of $\mathbb{R}^{2}$ the perimeters $\omega_{\partial}^{\prime}$ of the classes generate an inf-modular energy $\omega_{\partial}$ on the partial partitions.

\subsection{Lagrange families}

Definition 2. A scalar Lagrange family of energies $\left\{\omega(\lambda)=\omega_{\varphi}+\lambda \omega_{\partial}, \lambda \in \overline{\mathbb{R}}\right\}$ is one where $\omega(\lambda), \omega_{\varphi}$, and $\omega_{\partial}$ are singular and h-increasing, and further $\omega_{\partial}$ is inf-modular. ${ }^{4}$

Theorem 4. Let $\left\{\omega(\lambda)=\omega_{\varphi}+\lambda \omega_{\partial}\right\}$, be a scalar Lagrange family of energies on the partial partitions of a space $E$, and suppose $\lambda>0$. Given a braid $B$ on space $E$, let $\Pi_{\omega(\lambda)}, \Pi_{\omega_{\varphi}}$ and $\Pi_{\omega_{\partial}}$ be energetic lattices over the cuts $\pi \in \Pi(E, B)$ w.r.t. the Lagrangian $\omega(\pi, \lambda)$, the objective $\omega_{\varphi}$, and constraint $\omega_{\partial}$ respectively. The minimal element of $\Pi_{\omega(\lambda)}$ is denoted by $\pi^{*}(\lambda)$.

$$
0 \leq \lambda \leq \mu \quad \Rightarrow \quad \pi^{*}(\lambda) \succeq_{\omega_{\partial}} \pi^{*}(\mu) \text { and } \pi^{*}(\lambda) \preceq_{\omega_{\varphi}} \pi^{*}(\mu)
$$

i.e. as $\lambda$ increases, the sequence $\left\{\pi^{*}(\lambda), \lambda>0\right\}$ of the $\lambda$-cuts w.r.t. the $\Pi_{\omega(\lambda)}$ decreases in the energetic lattice $\Pi_{\omega_{\partial}}$ and increases in the energetic lattice $\Pi_{\omega_{\varphi}}$. Concerning the energies $\omega_{\partial}$ (resp. $\omega_{\varphi}$ ) we have:

$$
\lambda \leq \mu \Rightarrow \omega_{\partial}\left(\pi^{*}(\lambda)\right) \geq \omega_{\partial}\left(\pi^{*}(\mu)\right) \quad\left(\text { resp. } \omega_{\varphi}\left(\pi^{*}(\lambda)\right) \leq \omega_{\varphi}\left(\pi^{*}(\mu)\right)\right) .
$$

Proof for this theorem is given in [8]. The two energies $\omega_{\varphi}$ and $\omega_{\partial}$ vary in opposite senses on the minimal cuts. The relation in (9) generalizes the result of Salembier and Guigues over hierarchies and for linear energies $\omega_{\varphi}$ and $\omega_{\partial}$, to braids and Lagrange families. But the stronger implications (8) require the energetic lattices $\Pi_{\omega_{\varphi}}$ and $\Pi_{\omega_{\curvearrowright}}$. The role of inf-modularity of the constraint function $\omega_{\partial}$ is demonstrated in Theorem 4.

\footnotetext{
${ }^{4}$ one can write a vectorial version!
} 


\section{Lagrange minimization by energy (LME)}

In Everett's theorem one considers a set $X$ and objective $f$ and constraints $g, h$ defined at any point $x \in X$. Our situation slightly differs: the set $X$ is replaced by the set of all cuts of a braid $B$, and this set is equipped with three different lattice structures $\Pi_{\omega_{\varphi}}, \Pi_{\omega_{\partial}}$ and $\Pi_{\omega(\lambda)}$, governed by the three energies $\omega_{\varphi}, \omega_{\partial}$, and $\omega(\lambda)$. The primal and dual problems must be re-stated in the new framework of energetic lattice:

Problem 1. (LME Primal problem): Given a braid B, a constraint value $C$, and objective and constraint functions $\omega_{\varphi}$ and $\omega_{\partial}$, find the cut(s) $\pi \in B$ that minimize $\omega_{\varphi}(\pi)$, subject to the constraint $\omega_{\partial}(\pi) \leq C$.

The domain of the feasible cuts is $\Pi^{\prime} \subseteq \Pi(E, B)$

$$
\Pi^{\prime}=\left\{\pi, \pi \in \Pi(E, B), \omega_{\partial}(\pi) \leq C\right\}
$$

which by its definition is a braid of partitions itself [9]. In the Lagrangian lattice $\Pi_{\omega(\lambda)}(E, B)$ of energy $\omega(\lambda)=\omega_{\varphi}+\lambda \omega_{\partial}$, the $\lambda$-cut $\pi^{*}(\lambda)$ is a cut with least energy given the multiplier $\lambda$ for the Lagrangian energy $\omega\left(\pi^{*}(\lambda)\right)$. The energy $\omega\left(\pi^{*}(\lambda)\right)$ is a function of $\lambda, \omega_{\varphi}$ and $\omega_{\partial}$, but not of the cuts $\pi \in \Pi$. The dual problem is relative to this energy $\omega\left(\pi^{*}(\lambda)\right)$ :

Problem 2. (Multiplier Problem) Given a braid $B$ on $E$ and two energies $\omega_{\varphi}$ and $\omega_{\partial}$, find the parameter $\lambda$ which maximizes $\omega\left(\pi^{*}(\lambda)\right)$, subject to the constraint $\lambda>0$.

The following theorem answers both primal and dual problems (proof in [8])

Theorem 5. Given a braid $B$, let $\left\{\omega(\lambda)=\omega_{\varphi}+\lambda \omega_{\partial}, \lambda \in \overline{\mathbb{R}}\right\}$ be a scalar Lagrange family of energies. Let $\lambda^{*}=\inf \left\{\lambda \mid \omega_{\partial}\left(\pi^{*}(\lambda)\right) \leq C\right\}$. If

1. the feasible set $\Pi^{\prime}$ is not empty,

2. Multiplier-Constraint map: $\omega_{\partial}\left(\pi^{*}\left(\lambda^{*}\right)\right)-C=0$,

then $\pi^{*}\left(\lambda^{*}\right)$ and $\lambda^{*}$ are solutions of the problems 1 and 2 respectively. When $\omega_{\varphi}$ is strictly $h$-increasing, then the solution $\pi^{*}\left(\lambda^{*}\right)$ is unique.

Multiplier-Constraint Mapping: Theorem 5 demonstrates the multiplier dependence of the constraint function as already discussed by Everett's theorem 3. This Condition 2 in theorem (5) requires that for any constraint function value $\omega_{\partial}(\pi)=C$ there exists a corresponding optimal multiplier $\lambda^{*}$ such that $\omega_{\partial}\left(\pi\left(\lambda^{*}\right)\right)$. This is a highly unrealistic constraint. Furthermore finding an optimal multiplier in the dual domain returns us back to a combinatorial problem. Even for very simple constraint values, in the counter example 1 there are cases where no multiplier exists for an imposed constraint function value $C$. Instead these methods, including Guigues, Salembier and other, rely on the capability to approximate the imposed constraint function value by searching for a "good" value of multiplier $\lambda$. 


\section{Lagrange minimization by cut-Constraints (LMCC)}

In the LME model, the constraint function $\omega_{\partial}$ and the $\operatorname{cost} C$ are numerical, while the minimization itself is expressed in the energetic lattices $\Pi_{\omega_{\varphi}}$ and $\Pi_{\omega(\lambda)}$. Can we, alternatively, reformulate the constraint conditions directly with the cuts? We now examine this question, by looking for the cuts smaller than or equal to a given cut $\pi_{C} \in \Pi_{\omega_{\partial}}$ :

Problem 3. Find minimal cut $\pi_{\varphi} \in \Pi_{\omega_{\varphi}}$ subject to the constraint $\pi_{\varphi} \preceq_{\omega_{\partial}} \pi_{C}$.

$$
\Pi_{C}=\left\{\pi \mid \pi \in \Pi, \pi \preceq \omega_{\partial} \pi_{C}\right\}
$$

Here $\pi_{C}$ is the set of feasible solutions and we have $\pi_{\varphi}=\curlywedge_{\omega_{\varphi}}\left\{\pi \mid \pi \in \Pi_{C}\right\}$. As before, the Lagrangian $\omega(\lambda)=\omega_{\varphi}+\lambda \omega_{\partial}, \lambda \in \overline{\mathbb{R}}$, is introduced. It induces the energetic lattice $\Pi_{\omega(\lambda)}$ of minimal cut $\pi^{*}(\lambda)$. A new minimal $\lambda$ is also introduced by

$$
\lambda^{*}=\sup \left\{\lambda \mid \pi^{*}(\lambda) \preceq \omega_{\partial} \pi_{C}\right\}
$$

Problem 4. LMCC multiplier problem: find the value of the parameter $\lambda$ which optimizes $\pi^{*}(\lambda)$ in $\Pi\left(\omega_{\partial}\right)$ subject to the constraint $\lambda \geq 0$.

For solving jointly both problems 3 and 4, the following conditions are needed:

Theorem 6. Given a braid $B$, let $\left\{\omega(\lambda)=\omega_{\varphi}+\lambda \omega_{\partial}, \lambda \in \overline{\mathbb{R}}\right\}$ be a scalar Lagrange family of energies. Let $\lambda^{*}=\sup \left\{\lambda \mid \pi^{*}(\lambda) \preceq \omega_{\partial} \pi_{C}\right\}$. If

1. Constraint satisfaction: the set $\Pi_{C}=\left\{\pi \mid \pi \in \Pi, \pi \preceq \omega_{\partial} \pi_{C}\right\}$ is not empty,

2. Positive multiplier: $\lambda \geq 0$,

3. Energetic-Lattice constraint assumption: $\pi_{\omega_{\varphi}} \succeq_{\omega_{\varphi}} \pi^{*}\left(\lambda^{*}\right)$.

are fulfilled, then the set of feasibility in $\lambda$ is $\lambda \geq \lambda^{*}$, and $\pi^{*}\left(\lambda^{*}\right)$ and $\lambda^{*}$ are the unique solutions to the problems 3 and 4 respectively.

Proof. We first prove that the set of feasible $\lambda$ are $\lambda \geq \lambda^{*}$. According to relation 11 when $\lambda>\lambda^{*}$, then $\pi^{*}(\lambda)$ doest not belong to the feasible set $\Pi_{C}$. Therefore, if $\lambda$ is such that $\pi^{*}(\lambda) \preceq \omega_{\partial} \pi_{C}$, then $\lambda \leq \lambda^{*}$; if in addition $\lambda \geq 0$, then theorem 4 applies and $\pi^{*}\left(\lambda^{*}\right) \leq \pi^{*}(\lambda)$, hence $\pi^{*}\left(\lambda^{*}\right) \preceq \omega_{\partial} \pi_{C}$, i.e. $\pi^{*}\left(\lambda^{*}\right) \in \Pi_{C}$. Consequently $\pi^{*}\left(\lambda^{*}\right) \succeq_{\omega_{\varphi}} \pi\left(\omega_{\varphi}\right)$, and by assumption $3, \pi_{\varphi}=\pi^{*}\left(\lambda^{*}\right)$. The minimal cut (of Lattice $\Pi_{\omega\left(\lambda^{*}\right)}$ is a solution of problem 3 , and even the unique one, since $\pi_{\varphi}$ is the minimal element of a lattice. Concerning the multiplier problem we can apply Theorem 4 since $\lambda \geq 0$, which gives:

$$
\Upsilon_{\omega_{\partial}} \pi^{*}(\lambda) \preceq \omega_{\partial} \pi^{*}\left(\lambda^{*}\right), \forall \lambda \geq \lambda^{*} \geq 0 \text {. }
$$

As $\pi^{*}\left(\lambda^{*}\right)$ is also an element of left hand side of the above inequality, thus we obtain equality which solves the the multiplier problem. 


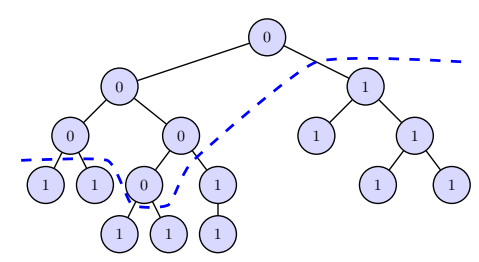

Fig. 3. Constraint cut $\pi_{0}$ shown in dotted line, which takes all classes below it. The 1-classes have $\omega_{\partial}>C$, the 0 -classes have $\omega_{\partial} \leq C$. First index the classes of $H$ by a lexicographic ordering from the root $E$ to the leaves, and go top-down beginning at the root. When a class $S$ has all its sons $T$ such that $\omega_{\partial}(T) \leq C$, then replace $S$ by its sons. We assume for that the leaves in the hierarchy of partitions must all satisfy the constraint to ensure a non-void feasible set.

In LMCC the minimal cut is unique, even when the energy is not strictly h-increasing. This authorizes the use of sup and inf composed energies. The comparison between LME and LMCC frameworks is instructive. One can also notice that the assumption 3 of the theorem 6 turns out to be weaker than the corresponding notion of in Theorem 5 , as it involves an inequality only. Both models show that Lagrangians still work for lattices of cuts, and not just only on numerical lattices of energies. An interesting feature is that Theorem 6 applies for infinite partitions of $E$, as soon as the number of classes is locally finite. This covers the "remote sensing type" of situations, where the zone under study is incomparably smaller than the total extension of the scene. The LMCC works since it avoids the constraint approximation problem.

\section{Class Constrained Minimization (CCM)}

We now study a constraint applied purely to classes. This restricts the constraint function to be defined now on the classes and no more on the partitions. This section treats firstly the case of hierarchies and then that of braids, and develops an alternative method for constrained optimization, which does not resort to Lagrangians.

The hierarchy $H$ under study is supposed to be finite. Provide the classes $S \in \mathcal{S}$ of the hierarchy $H$ with an energy $\omega_{\partial}$ and fix a constraint value $C$. Introduce now an objective energy $\omega_{\varphi}$ that is $h$-increasing and singular. We can now set the following problem:

Problem 5. Find the cut(s) of $H$ of smallest energy $\omega_{\varphi}(\pi)$, such that all classes in these cuts satisfy the constraint $\omega_{\partial}(S) \leq C$.

The method consists in generating a new hierarchy $H^{\prime}$ where the minimization of $\omega_{\varphi}$ is no longer conditioned. Let $\mathcal{A}(C)$ stand for the family of the cuts $\pi$ of $H$ whose energies of all classes are $\leq C$ :

$$
\pi \in \mathcal{A}(C) \Leftrightarrow \quad\left\{S \sqsubseteq \pi \Rightarrow \omega_{\partial}(S) \leq C\right\} .
$$


Obviously, the problem is feasible if and only if $\mathcal{A}(C)$ is not empty. Since the family $\mathcal{A}(C)$ is closed under the refinement infimum, it admits a smallest element $\pi_{0}$ :

$$
\pi_{0}=\wedge\{\pi, \pi \in \mathcal{A}(C)\}
$$

The classes of $\pi_{0}$ can be interpreted as the set of leaves of a new hierarchy $H^{\prime}$, identical to $H$ above and on $\pi_{0}$, but where all classes below $\pi_{0}$ are removed (see Figure 3). The cuts $\pi$ of $H^{\prime}$ are exactly those of $H$ that satisfy the constraint $\omega_{\partial}(\pi) \leq C$. The problem now reduces to find the minimal cut of $H^{\prime}$ w.r.t. $\omega_{\varphi}$, a question that we already know how to treat. As the minimization is understood in the $\omega_{\varphi}$-energetic lattice $\Pi\left(\omega_{\varphi}, H^{\prime}\right)$ relative to $H^{\prime}$, we have to suppose $\omega_{\varphi}$ singular and $h$-increasing, and we can state:

Proposition 1. When $\omega_{\varphi}$ is a singular and h-increasing energy, then the minimal cut $\pi_{\varphi}^{*}$ in the $\omega_{\varphi}$-energetic lattice $\Pi\left(\omega_{\varphi}, H^{\prime}\right)$ is also a cut of smallest $\omega_{\varphi}$ energy in $\Pi\left(\omega_{\varphi}, H\right)$ whose all classes $S^{*}$ satisfy the cost constraint $\omega_{\partial}\left(S^{*}\right) \leq C$.

The result is important. It grants the existence and the uniqueness of the minimal cut $\pi_{\varphi}^{*}$ under very large conditions: no prerequisite is needed for $\omega_{\partial}$, and uniquely singularity and $h$-increasingness for $\omega_{\varphi}$. Note that the cost $C$ need not be constant. Equivalence (12) holds on each class separately. $C$ may vary through the space, or according to the level $i$ in the hierarchy. When the energy $\omega_{\varphi}$ is also increasing w.r.t. the refinement of the cuts (e.g. the usual version of Mumford-Shah objective energy), i.e. when:

$$
\pi_{1} \leq \pi_{2} \quad \Rightarrow \omega_{\varphi}\left(\pi_{1}\right) \leq \omega_{\varphi}\left(\pi_{2}\right),
$$

then the minimal cut $\pi_{\varphi}^{*}$ coincides with $\pi_{0}$, since $\pi_{0} \leq \pi \Rightarrow \omega_{\varphi}\left(\pi_{0}\right)=\wedge\left\{\omega_{\varphi}(\pi), \pi \in\right.$ $\mathcal{A}(C)\}=\omega_{\varphi}\left(\pi_{\varphi}^{*}\right)$. It remains to build up the hierarchy $H^{\prime}$ i.e. to find the leaves $\pi_{0}$. Suppose now that $\omega_{\partial}$ is inf-modular. Let

$$
\omega_{\partial}(S) \leq \wedge\left\{\omega_{\partial}(T), T \text { son of } S\right\},
$$

i.e. the energy $\omega_{\partial}$ of class $S$ is smaller or equal to the smallest energy of the sons of $S$. Such class inf-modularity acts on classes and no longer on p.p. as in Rel.(6), but both are equivalent. The partition $\pi_{0}$ is obtained at the end of the scan, i.e. in one pass. A toy example is given in Figure 3. W.r.t. the $\omega_{\partial}$-energetic lattice $\Pi_{\omega_{\partial}, H^{\prime}}$, the cut $\pi_{0}$ turns out to be a maximum.

\section{Conclusion}

We began this theoretical paper by using the Everett's theorem to show that $\lambda$ cuts in case of Guigues [6], and optimal prunings of Salembier [11] provide only an upper-bound on minimal objective energy. This was explicated further by the dependence of the constraint function values on the Lagrangian multiplier, and also the possibility of the non-existence of multipliers for certain constraint 
values and vice versa. The constraint function values were shown to be lattice structured and not varying continuously. This motivated the use of an energetic lattice framework. This gave us two ways to enforce of a constraint:

Numerical Minimization: Lagrangian Minimization by Energy (LME) model enforces a numerical constraint on energy, without referring to the partition structure. The Energetic lattice was used to generalize the Lagrangian model in LME, when one works in the space of partitions from a braid.

Lattice structured Minimization: Lagrange Minimization by Cut-Constraints (LMCC) and Class Constrained Minimization (CCM) models enforce the constraint in the form a partition. This does not involve any numerical constraint function, but one that is driven or evaluated on the energetic lattice.

\section{References}

1. Bach, F.: Learning with submodular functions: A convex optimization perspective. Foundations and Trends in Machine Learning 6(2-3), 145-373 (2013)

2. Ballester, C., Caselles, V., Igual, L., Garrido, L.: Level lines selection with variational models for segmentation and encoding. Journal of Mathematical Imaging and Vision 27(1), 5-27 (2007)

3. Boyd, S., Vandenberghe, L.: Convex Optimization. Cambridge University Press, New York, NY, USA (2004)

4. Breiman, L., Friedman, J.H., Olshen, R.A., Stone, C.J.: Classification and Regression Trees. Wadsworth (1984)

5. Everett, H.: Generalized Lagrange multiplier method for solving problems of optimum allocation of resources. Operations Research 11(3), 399-417 (1963)

6. Guigues, L., Cocquerez, J.P., Men, H.L.: Scale-sets image analysis. International Journal of Computer Vision 68(3), 289-317 (2006)

7. Kiran, B.R., Serra, J.: Global-local optimizations by hierarchical cuts and climbing energies. Pattern Recognition 47(1), 12 - 24 (2014)

8. Kiran, B.R.: Energetic Lattice based optimization. Ph.D. thesis, Université ParisEst, LIGM-A3SI, ESIEE (2014)

9. Kiran, B.R., Serra, J.: Braid of partitions. submitted to ISMM 2015 (2015)

10. Ronse, C.: Partial partitions, partial connections and connective segmentation. J. Math. Imaging Vis. 32(2), 97-125 (Oct 2008)

11. Salembier, P., Garrido, L.: Binary partition tree as an efficient representation for image processing, segmentation, and information retrieval. IEEE Trans. on Image Processing 9(4), 561-576 (2000)

12. Shoham, Y., Gersho, A.: Efficient bit allocation for an arbitrary set of quantizers. Acoustics, Speech \& Signal Processing, IEEE Transactions on 36(9), 1445-1453 (Sep 1988) 